\title{
Suppression of the shear Raman mode in defective bilayer $\mathrm{MoS}_{2}$
}

Cite as: J. Appl. Phys. 125, 064305 (2019); https://doi.org/10.1063/1.5086366

Submitted: 20 December 2018 . Accepted: 31 January 2019 . Published Online: 14 February 2019

Pierce Maguire (D), Clive Downing, Jakub Jadwiszczak (D), Maria O'Brien, Darragh Keane (D), John B. McManus (D), Georg S. Duesberg (D), Valeria Nicolosi, Niall McEvoy (D), and Hongzhou Zhang (iD)
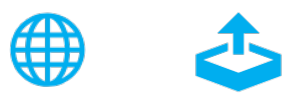

\section{ARTICLES YOU MAY BE INTERESTED IN}

Photoresponsivity enhancement in monolayer $\mathrm{MoS}_{2}$ by rapid $\mathrm{O}_{2}$ :Ar plasma treatment

Applied Physics Letters 114, 091103 (2019); https://doi.org/10.1063/1.5086726

Subsampled STEM-ptychography

Applied Physics Letters 113, 033104 (2018); https://doi.org/10.1063/1.5040496

SQUID-detected FMR: Resonance in single crystalline and polycrystalline yttrium iron garnet Review of Scientific Instruments 89, 044701 (2018); https://doi.org/10.1063/1.5009731

\section{Journal of Applied Physics Polymer-Grafted Nanoparticles}




\title{
Suppression of the shear Raman mode in defective bilayer $\mathrm{MoS}_{2}$
}

\author{
Cite as: J. Appl. Phys. 125, 064305 (2019); doi: 10.1063/1.5086366 \\ Submitted: 20 December 2018 . Accepted: 31 January 2019 . \\ Published Online: 14 February 2019
}

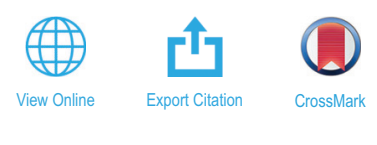

Pierce Maguire, ${ }^{1,2}$ (1) Clive Downing, ${ }^{2,3}$ Jakub Jadwiszczak, ${ }^{1,2}$ (D) Maria O'Brien, ${ }^{2,3}$ Darragh Keane, ${ }^{2,3}$ (D) John B. McManus, 2,3 (D) Georg S. Duesberg, ${ }^{2,3,4}$ (D) Valeria Nicolosi, ${ }^{2,3}$ Niall McEvoy, ${ }^{2,3}$ (D) and Hongzhou Zhang ${ }^{1,2, a)}$ (D)

\author{
AFFILIATIONS \\ ${ }^{1}$ School of Physics, Trinity College Dublin, Dublin 2, D02 PN40, Ireland \\ ${ }^{2}$ AMBER Centre, CRANN Institute, Trinity College Dublin, Dublin 2, D02 PN40, Ireland \\ ${ }^{3}$ School of Chemistry, Trinity College Dublin, Dublin 2, DO2 PN4O, Ireland \\ ${ }^{4}$ Institute of Physics, EIT 2, Faculty of Electrical Engineering and Information Technology, Universität der Bundeswehr München, \\ Werner-Heisenberg-Weg 39, 85577 Neubiberg, Germany
}

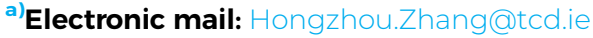

\begin{abstract}
We investigate the effects of lattice disorders on the low frequency Raman spectra of bilayer $\mathrm{MoS}_{2}$. The bilayer MoS $\mathrm{Mas}_{2}$ subjected to defect engineering by irradiation with a $30 \mathrm{keV} \mathrm{He}^{+}$ion beam, and the induced morphology change was characterized by transmission electron microscopy. When increasing the ion dose, the shear mode is observed to red-shift, and it is also suppressed sharply compared to other Raman peaks. We use the linear chain model to describe the changes to the Raman spectra. Our observations suggest that the crystallite size and orientation are the dominant factors behind the changes to the Raman spectra.
\end{abstract}

Published under license by AIP Publishing. https://doi.org/10.1063/1.5086366

\section{INTRODUCTION}

As research on transition metal dichalcogenides (TMDs) blooms, ${ }^{1-4}$ it is crucial to understand the interfacial dynamics of these layered semiconductors in the few-layer limit. Low-frequency interlayer vibrational Raman modes serve as a fingerprint of the interlayer interactions between van der Waals-bonded sheets in both homo- and heterostructures. ${ }^{5-8}$ The low-frequency Raman modes of $\mathrm{MoS}_{2}$ include the in-plane shear mode (SM) and the out-of-plane layer breathing mode (LBM). Layer thickness, stacking order, and angular lattice mismatch can be directly probed in vertically-assembled heterostructures of TMDs by investigating changes to these modes. ${ }^{6-9-13}$ On the other hand, Raman spectroscopy has remained a high-throughput and non-destructive way of analyzing defects in $\mathrm{MoS}_{2}$ flakes in the last decade. In particular, the $E_{2 g}^{1}\left(\sim 385 \mathrm{~cm}^{-1}\right)$ and $A_{1 g}\left(\sim 403 \mathrm{~cm}^{-1}\right)$ modes are routinely used to identify layer thickness in exfoliated and deposited $\mathrm{MoS}_{2}$ samples. ${ }^{10,14-17}$ Relative changes in intensities, positions, or widths are used to characterise the lattice modification of
$\mathrm{MoS}_{2} \cdot{ }^{18-21}$ However, the effects of lattice defects on the lowfrequency $\left(<50 \mathrm{~cm}^{-1}\right)$ modes of few-layer $\mathrm{MoS}_{2}$ have not been explored, even while low-frequency Raman spectroscopy should play a vital role in the characterization of interfacial dynamics of defective layered semiconductors.

Herein, we analyze the low-frequency modes of the Raman spectrum of bilayer $\mathrm{MoS}_{2}$ as a function of increasing disorder introduced by $\mathrm{He}^{+}$irradiation at a beam energy of $30 \mathrm{keV}$. The helium ion microscope has been established as a tool for site-specific modification of 2D materials. ${ }^{18,22-27}$ It has been used to precisely introduce defects, ${ }^{21}$ fabricate nanoribbons, and periodic arrays of nanodots, ${ }^{18,22}$ and introduce doping on the nanoscale. ${ }^{23}$

\section{EXPERIMENTAL METHODS}

$\mathrm{MoS}_{2}$ was prepared using a previously reported chemical vapor deposition (CVD) technique. ${ }^{28}$ Bilayer flakes of $\mathrm{MoS}_{2}$ were identified on the $\mathrm{SiO}_{2}$ surface by optical contrast and Raman spectroscopy. ${ }^{14,29}$ The Zeiss ORION NanoFab microscope was 
used to irradiate arrays of $4 \times 4 \mu \mathrm{m}^{2}$ regions in $\mathrm{MoS}_{2}$ with $\mathrm{He}^{+}$ at an energy of $30 \mathrm{keV}$ and an angle of incidence of $0^{\circ}$. These regions received doses ranging from $8 \times 10^{13}$ to $2 \times 10^{16}$ $\mathrm{He}^{+} \mathrm{cm}^{-2}$. Further details of the ion irradiation are provided in the supplementary material. Ex situ Raman and PL spectroscopy was carried out using a WITec Alpha 300R system (532 $\mathrm{nm}$ laser) with a 1800 lines/mm diffraction grating and a $100 \times$ objective (NA $=0.95)$ (spot size $\sim 0.3 \mu \mathrm{m}$ ). The instrument was equipped with a RayShield Coupler to detect the Raman signal close to the Rayleigh line. The laser power was $\sim 170 \mu \mathrm{W}$ or less to minimise damage to the samples. Raman and PL maps were generated by taking four spectra per $\mu \mathrm{m}$ in both the $\mathrm{x}$ and $\mathrm{y}$ directions over large areas. The acquisition time for each spectrum was $0.113 \mathrm{~s}$. The spectra from each irradiated region were acquired by averaging. Raman, PL, and optical images are shown in Fig. S1 in the supplementary material. Lorentzian distributions were fitted to the Raman peaks as demonstrated in Fig. S4 in the supplementary material.

\section{RESULTS AND DISCUSSION}

Figure 1 shows representative Raman spectra. In the low frequency region [Fig. 1(a)], the SM and LBM peaks are centred at $\sim 23 \mathrm{~cm}^{-1}$ and $\sim 41 \mathrm{~cm}^{-1}$, respectively, which are in excellent agreement with the reported values for bilayer $\mathrm{MoS}_{2} \cdot{ }^{9,17,30}$ For an increasing dose, the SM is clearly observed to red-shift and broaden while the LBM does not appear to change. At higher doses $\left(\sim 1 \times 10^{16} \mathrm{He}^{+} \mathrm{cm}^{-2}\right)$, the low frequency modes become indistinguishable from noise. In the high frequency region [Fig. 1(b)], the characteristic $E_{g}$ and $A_{1 g}$ peaks of bilayer $\mathrm{MoS}_{2}$ are found at $384 \mathrm{~cm}^{-1}$ and $406.6 \mathrm{~cm}^{-1}$, respectively, thus exhibiting a high frequency peak separation of $\sim 22.6 \mathrm{~cm}^{-1}$ typical of CVD bilayer $\mathrm{MoS}_{2} .{ }^{14,31}$ With an increasing ion dose, the $A_{1 g}$ peak is observed to broaden, while

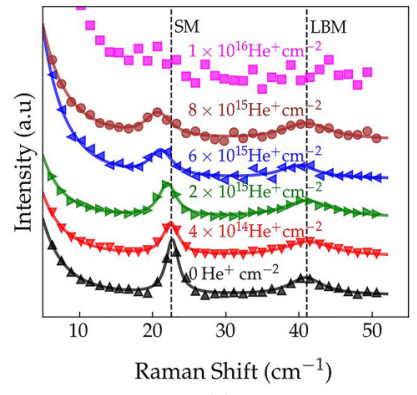

(a)

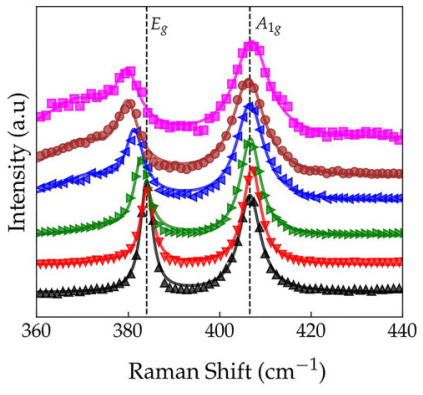

(b)
FIG. 1. A representative selection of Raman spectra of bilayer $\mathrm{MoS}_{2}$ excited by a $532 \mathrm{~nm}$ laser after irradiation with $\mathrm{He}^{+}$at $30 \mathrm{keV}$ with a $0^{\circ}$ angle of incidence. The evolution of the spectra with increased ion dose is shown ascending from the bottom in black to the top in magenta. (a) shows the low frequency region in which the SM and LBM peaks are labelled. (b) shows the high frequency region in which the $E_{g}$ and $A_{1 g}$ peaks are labelled. In both high and low frequency ranges, each spectrum is normalized to its $A_{1 g}$ peak. The intensity is rescaled between high and low frequency spectra for maximum visibility. the $E_{g}$ peak broadens, splits and is shifted downward in energy as has been reported before in similar conditions..$^{21,32,33}$

Figure 2 shows photoluminescence (PL) spectra acquired from the same regions of bilayer $\mathrm{MoS}_{2}$. We observe peaks at $\sim 1.85 \mathrm{eV}$ and $\sim 2.01 \mathrm{eV}$, corresponding to A and B direct excitonic transitions, respectively. ${ }^{34}$ The increased disorder is strongly associated with a lower quantum efficiency. The decrease in the A exciton peak intensity is accompanied by a blue-shift which is attributed to strain caused by ion-induced defects. ${ }^{35}$ Above a dose of $\sim 2 \times 10^{15} \mathrm{He}^{+} \mathrm{cm}^{-2}$, we can no longer observe a clearly defined emission peak.

Figures 3(a) and 3(b) show the position and width, respectively, of the low frequency Raman peaks as a function of the ion dose (analysis of the high frequency peaks is presented in Fig. S3 in the supplementary material). The SM clearly shifts downward in position as it broadens. However, the LBM does not appear to change systematically in position or width. The measured SM frequency, $\omega$, is given by

$$
\omega=\omega_{0}-2.04 \times 10^{-8} \sqrt{\mathrm{S}},
$$

where $\omega_{0}=22.8 \mathrm{~cm}^{-1}$ is the frequency of the non-irradiated bilayer $\mathrm{MoS}_{2}$ and $\mathrm{S}$ is the ion dose $\left(\mathrm{He}^{+} \mathrm{cm}^{-2}\right)$. The measured width, $\Gamma$, is given by

$$
\Gamma=\Gamma_{0}+0.247 \sqrt{S}
$$

where $\Gamma_{0}=1.9 \mathrm{~cm}^{-1}$ is the width of the undamaged bilayer $\mathrm{MoS}_{2}$.

Figure 3(c) shows the heights of the low frequency modes, $\mathrm{I}(\mathrm{X})$, where $\mathrm{X}=\mathrm{SM}$ or LBM, normalized to the respective $A_{1 g}$ intensity, $I\left(A_{1 g}\right)$. While the intensity of $I(L B M) / I\left(A_{1 g}\right)$ is virtually constant, $\mathrm{I}(\mathrm{SM}) / \mathrm{I}\left(\mathrm{A}_{1 g}\right)$ is observed to diminish with an increasing dose. The SM is clearly suppressed more rapidly than other peaks by increasing disorder. The SM intensity ratio varies with the dose $\mathrm{I}(\mathrm{SM}) \propto \mathrm{I}\left(\mathrm{A}_{1 g}\right) / \mathrm{S}^{d}$, where $d=0.17$ is a fitting parameter.

Figure 4(a) is a high resolution transmission electron microscopy (HRTEM) image of mechanically exfoliated bilayer $\mathrm{MoS}_{2}$ showing clearly defined layers with uniform interlayer spacing (see Fig. S5 in the supplementary material for TEM sample preparation). Above and below the $\mathrm{MoS}_{2}$, the protective platinum layer and the $\mathrm{SiO}_{2}$ substrate are visible, respectively. Figure 4(b) shows a region of the same sample which has been irradiated with $6 \times 10^{15} \mathrm{He}^{+} \mathrm{cm}^{-2}$ which is in the middle-to-high part of the range of doses used in our spectroscopic experiments. An additional amorphous hydrocarbon layer of helium ion beam-induced deposition (IBID) is visible on top of the $\mathrm{MoS}_{2}$. A clear increase in the surface asperity is noted after irradiation. In certain regions, $\mathrm{MoS}_{2}$ is still visibly present but separate layers cannot be resolved. This may suggest amorphization or local twisting of the layers such that they are imaged off-axis.

Figure 4(c) is a histogram of interlayer separation evaluated from the two TEM images, using a fitting process 


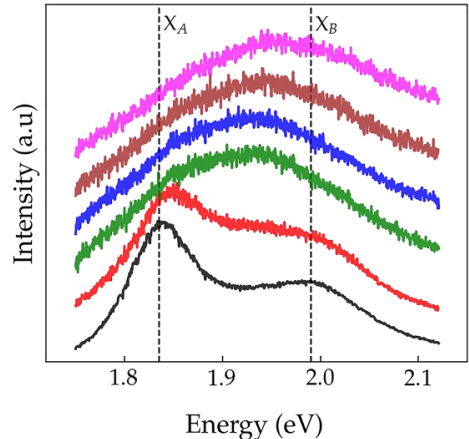

FIG. 2. PL spectra of the same regions with the same color-dose correspondence as in Fig. 1. $X_{A}$ and $X_{B}$ label the $A$ and $B$ excitons, respectively. The $A$ exciton emission is observed to decline rapidly with an increasing dose. The spectra are normalized to the maximum of each to allow comparison of the two peaks. The spectra are presented without normalisation in Fig. S2 in the supplementary material.

described in the supplementary material. There appears to be very little change in the mean layer separation after irradiation. However, the disordered distribution is clearly more diffuse and skewed toward higher values, reflected in the increased kurtosis and skewness, respectively.

A linear chain model (LCM) has been used to describe interlayer restoring forces by treating each layer as a rigid ball. ${ }^{17,36}$ The model has accurately predicted the frequencies of the low frequency modes in pristine $\mathrm{MoS}_{2}$ as a function of layer number. The single layer mass per unit area $\left(\mu_{0}\right)$ of pristine $\mathrm{MoS}_{2}$ is $3.03 \times 10^{-26} \mathrm{~kg} \AA^{-2}$. Since sputtering of specimen atoms is caused by the ion irradiation, the effects are accounted for in $\mu$ by applying an approximate correction related to the ion dose. However, as demonstrated in Figs. S8 and S9 in the supplementary material, this effect would lead

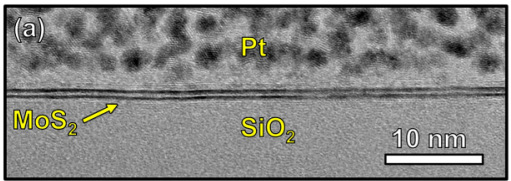

(a)

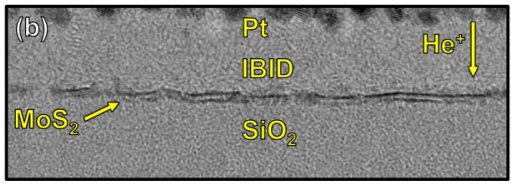

(b)

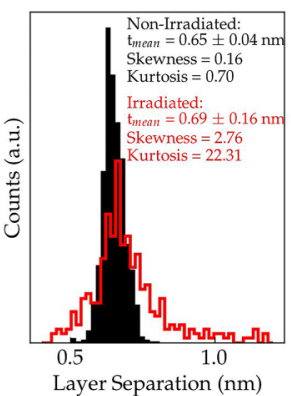

(c)

FIG. 4. Cross-sectional HRTEM images and analysis of bilayer $\mathrm{MoS}_{2}$. (a) and (b) are images of a non-irradiated region and a region irradiated with $6 \times 10^{15} \mathrm{He}^{+} \mathrm{cm}^{-2}$ respectively. Both (a) and (b) have the same scale. The labels and arrows indicate the various materials and the direction of the ion beam irradiation. (c) is a histogram of the interlayer separation for nonirradiated and irradiated $\mathrm{MoS}_{2}$. The filled black bars represent the layer separation of the non-irradiated region [from Fig. 4(a)], and the unfilled red bars represent the layer separation of the irradiated region [from Fig. 4(b)]. The mean, skewness, and kurtosis of each distribution are presented in the respective colors.

to a very small blue shift which by calculation and experiment is indicated to be negligible.

Given the dose, $\mathrm{S}$, and the sputter yield, $\gamma(=0.007$ from the previous reports $\left.{ }^{21,37,38}\right)$, then $\mu(\mathrm{S})$ for irradiated $\mathrm{MoS}_{2}$ is given by $\mu(\mathrm{S})=\mu_{0}(1-\gamma \mathrm{S} / n)$, where $n$ is the atomic density. This gives the sputtering-corrected LCM as

$$
\omega(\mathrm{S})=\frac{1}{\sqrt{2} \pi \mathrm{c}} \sqrt{\frac{\alpha}{\mu_{0}\left(1-\frac{\gamma \mathrm{S}}{n}\right)}} \sqrt{1+\cos \left(\frac{\pi}{\mathrm{N}}\right)},
$$

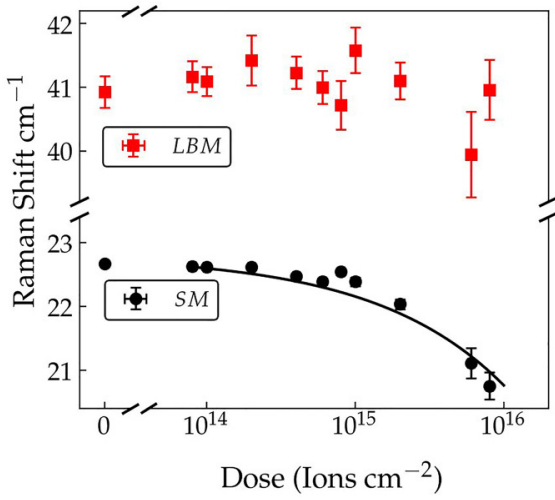

(a)

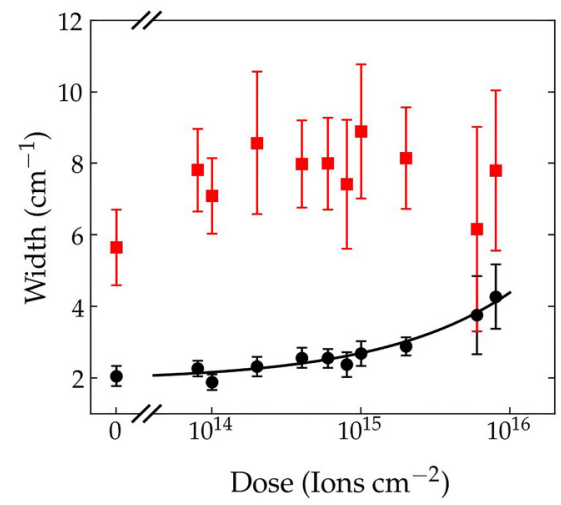

(b)

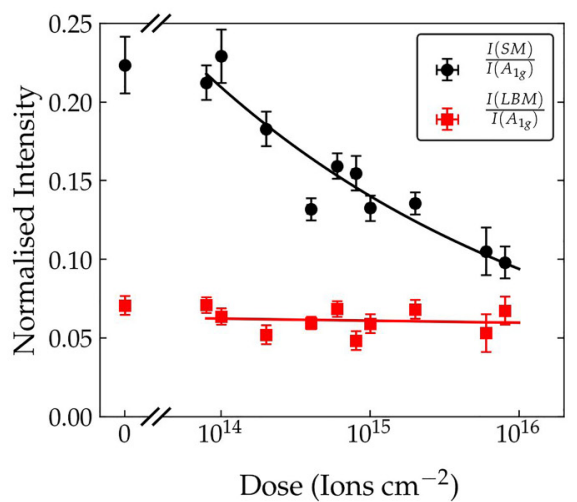

(c)

FIG. 3. Evolution of the low frequency Raman modes as a function of ion dose. Error bars are from the fitting error. (a) shows the change in frequency of the two low frequency modes. The shear mode frequency is fitted with Eq. (1). (b) shows the change in width of the two low frequency modes. The shear mode position is fitted with Eq. (2). (c) shows the ratio of the intensity of the two low frequency modes to the $A_{1 g}$ peak. The shear mode intensity ratio is fitted as described in the main text. 


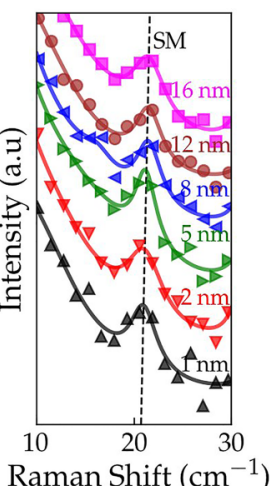

(a)

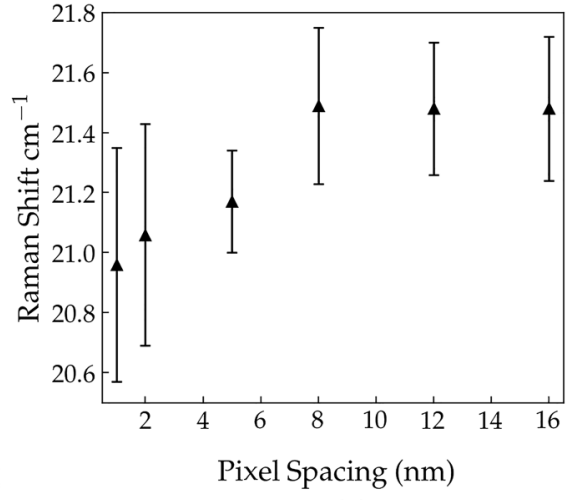

(b)
FIG. 5. Evolution of the SM as a function of ion pixel spacing at a fixed dose of $6 \times 10^{15} \mathrm{~cm}^{-2}$ and with a probe size of $\sim 4 \mathrm{~nm}$. (a) shows the SM with the different pixel spacings labelled. (b) shows the change in position of the SM as a function of the irradiation pixel spacing

where $c$ is the speed of light in $\mathrm{cm} \mathrm{s}^{-1}, \mathrm{~N}$ is the layer number (fixed at 2 in this work), $\alpha$ is the interlayer force constant per unit area which is related to the interlayer separation, $t$, and the shear modulus, $\mathrm{C}$ by $\alpha=\mathrm{C} / \mathrm{t}$. The shear modulus has been reported as $\mathrm{C} \simeq 17.9 \mathrm{GPa} .{ }^{17}$ For $\mathrm{S}=0$, the shear mode position is calculated to be $23.2 \mathrm{~cm}^{-1}$ which is consistent with our experimental value of $22.8 \mathrm{~cm}^{-1}$. The separation of two nonirradiated layers in bilayer $\mathrm{MoS}_{2}$ measured from our TEM results is $t=0.65 \pm 0.04 \mathrm{~nm}$, in good agreement with the literature. ${ }^{17}$ In the absence of other effects, the separation that would be required to explain the red shift is $\sim 0.76 \mathrm{~nm}$ (see Fig. S9 in the supplementary material). This is not consistent with the TEM results, so we cannot attribute the red shift to a change in separation.

The two key findings of our Raman spectroscopy experiments clear from Figs. 1 and 2 are related to the shear mode, i.e., the drop in the normalized intensity $(\sim 2.3 \times)$ and the frequency red-shift $\left(\Delta \omega \simeq 1.8 \mathrm{~cm}^{-1}\right)$ with ion irradiation. Small twisting angles can introduce a periodicity mismatch between layers, altering the stacking configuration and causing a sharp decay of SM intensity and a downward shift in position. ${ }^{39,40}$ The twisting is evident in the TEM observation (Fig. 4). However, the twisting alone cannot explain the observed peak shift (see Fig. S10 in the supplementary material). Reductions in the shear modulus also contribute to a redshift in the SM. This is due to the reduced crystallinity in $\mathrm{MoS}_{2}$ under ion irradiation. The reduced crystallinity leads to better lubricating properties, attributed to a greater tendency to exfoliate and therefore a reduced shear modulus. ${ }^{41}$ To further corroborate this, we studied the effect of varying the pixel spacing on the SM with a new set of irradiation experiments. We used a fixed dose of $6 \times 10^{15} \mathrm{~cm}^{-2}$ but we varied the pixel spacing from 1 to $16 \mathrm{~nm}$. The probe size was $\sim 4 \mathrm{~nm}$ (see Fig. S7 in the supplementary material). ${ }^{42}$ The resulting Raman spectra are presented in Fig. 5(a). Figure 5(b) shows that as the pixel spacing decreases, the red-shift of the SM is observed to increase (the total effect is approximately $0.5 \mathrm{~cm}^{-1}$ ). As pixel spacings decrease, the total undamaged area is reduced. This suggests that regions of defective $\mathrm{MoS}_{2}$ may have a reduced shear modulus in addition to the twisting effects.

\section{CONCLUSION}

In conclusion, we explored the effects of increased disorder on the low and high frequency Raman peaks of bilayer $\mathrm{MoS}_{2}$. In the low frequency range, we noticed that the shear mode red-shifted and dropped in intensity with an increased irradiation dose. We used TEM imaging to investigate and we suggest that these changes can be attributed to a mixture of local twisting of layers and a decline in the shear modulus due to reduced crystallite sizes.

\section{SUPPLEMENTARY MATERIAL}

See the supplementary material for further information on the irradiation, Raman, PL, and TEM experiments, data treatment, and layer separation calculations.

\section{ACKNOWLEDGMENTS}

The authors thank the staff at the Advanced Microscopy Laboratory (AML), CRANN, Trinity College Dublin. We acknowledge support from the following grants: Science Foundation Ireland (Grant Nos. 12/RC/2278, 15/SIRG/3329, 11/PI/1105, 07/SK/I1220a, 15/IA/3131, and 08/CE/I1432) and Irish Research Council (Grant No. GOIPG/2014/972).

\section{REFERENCES}

${ }^{1}$ K. S. Novoselov, A. Mishchenko, A. Carvalho, and A. H. Castro Neto, Science 353, aac9439 (2016).

${ }^{2}$ D. Jariwala, T. J. Marks, and M. C. Hersam, Nat. Mater. 16, 170 (2016).

${ }^{3}$ K. Kang, K. H. Lee, Y. Han, H. Gao, S. Xie, D. A. Muller, and J. Park, Nature 550, 229 (2017).

${ }^{4} J$. H. Sung, H. Heo, S. Si, Y. H. Kim, H. R. Noh, K. Song, J. Kim, C. S. Lee, S. Y. Seo, D. H. Kim, H. K. Kim, H. W. Yeom, T. H. Kim, S. Choi, J. S. Kim, and M. H. Jo, Nat. Nanotechnol. 12, 1064 (2017).

${ }^{\mathbf{5}}$ M. Boukhicha, M. Calandra, M. A. Measson, O. Lancry, and A. Shukla, Phys. Rev. B 87, 1 (2013).

${ }^{6}$ C. H. Lui, Z. Ye, C. Ji, K. C. Chiu, C. T. Chou, T. I. Andersen, C. Means-Shively, H. Anderson, J. M. Wu, T. Kidd, Y. H. Lee, and R. He, Phys. Rev. B 91, 1 (2015).

${ }^{7}$ J. Zhang, J. Wang, P. Chen, Y. Sun, S. Wu, Z. Jia, X. Lu, H. Yu, W. Chen, J. Zhu, G. Xie, R. Yang, D. Shi, X. Xu, J. Xiang, K. Liu, and G. Zhang, Adv. Mater. 28, 1950 (2016)

${ }^{8}$ Y. Li, S. Lin, Y. S. Chui, and S. P. Lau, ECS J. Solid State Sci. Technol. 5, Q3033 (2016).

${ }^{9}$ Y. Zhao, X. Luo, H. Li, J. Zhang, P. T. Araujo, C. K. Gan, J. Wu, H. Zhang, S. Y. Quek, M. S. Dresselhaus, and Q. Xiong, Nano Lett. 13, 1007 (2013).

${ }^{10}$ X. Zhang, W. P. Han, J. B. Wu, S. Milana, Y. Lu, Q. Q. Li, A. C. Ferrari, and P. H. Tan, Phys. Rev. B 87, 115413 (2013).

${ }^{11}$ X. Zhang, X. F. Qiao, W. Shi, J. B. Wu, D. S. Jiang, and P. H. Tan, Chem. Soc. Rev. 44, 2757 (2015).

${ }^{12}$ Z. Lin, B. R. Carvalho, E. Kahn, R. Lv, R. Rao, H. Terrones, M. A. Pimenta, and M. Terrones, 2D Mater. 3, 022002 (2016).

${ }^{13}$ Q. J. Song, Q. H. Tan, X. Zhang, J. B. Wu, B. W. Sheng, Y. Wan, X. Q. Wang, L. Dai, and P. H. Tan, Phys. Rev. B 93, 115409 (2016). 
${ }^{14}$ C. Lee, H. Yan, L. E. Brus, T. F. Heinz, J. Hone, and S. Ryu, ACS Nano 4 , 2695 (2010).

${ }^{15} \mathrm{H}$. Li, Q. Zhang, C. C. R. Yap, B. K. Tay, T. H. T. Edwin, A. Olivier, and D. Baillargeat, Adv. Funct. Mater. 22, 1385 (2012).

${ }^{16} \mathrm{M}$. O'Brien, N. McEvoy, D. Hanlon, T. Hallam, J. N. Coleman, and G. S. Duesberg, Sci. Rep. 6, 19476 (2016).

${ }^{17}$ H. Zeng, B. Zhu, K. Liu, J. Fan, X. Cui, and Q. M. Zhang, Phys. Rev. B 86, 241301(R) (2012).

${ }^{18}$ D. S. Fox, Y. Zhou, P. Maguire, A. O'Neill, C. O'Coileáin, R. Gatensby, A. M. Glushenkov, T. Tao, G. S. Duesberg, I. V. Shvets, M. Abid, M. Abid, H. C. Wu, Y. Chen, J. N. Coleman, J. F. Donegan, and H. Zhang, Nano Lett. 15, 5307 (2015).

${ }^{19}$ T. Y. Ko, A. Jeong, W. Kim, J. Lee, Y. Kim, J. E. Lee, G. H. Ryu, K. Park, D. Kim, Z. Lee, M. H. Lee, C. Lee, and S. Ryu, 2D Mater. 4, 014003 (2016).

${ }^{20} \mathrm{~N}$. Choudhary, M. R. Islam, N. Kang, L. Tetard, Y. Jung, and S. I. Khondaker, J. Phys. Condens. Matter 28, 364002 (2016).

${ }^{21}$ P. Maguire, D. S. Fox, Y. Zhou, Q. Wang, M. O'Brien, J. Jadwiszczak, C. P. Cullen, J. B. McManus, S. Bateman, N. McEvoy, G. S. Duesberg, and H. Zhang, Phys. Rev. B 98, 134109 (2018).

${ }^{22}$ Y. Zhou, P. Maguire, J. Jadwiszczak, M. Muruganathan, H. Mizuta, and H. Zhang, Nanotechnology 27, 325302 (2016).

${ }^{23}$ G. Nanda, S. Goswami, K. Watanabe, T. Taniguchi, and P. F. A. Alkemade, Nano Lett. 15, 4006 (2015).

${ }^{24}$ V. Iberi, L. Liangbo, A. V. Ievlev, M. G. Stanford, M. W. Lin, X. Li, M. Mahjouri-Samani, S. Jesse, B. G. Sumpter, S. V. Kalinin, D. C. Joy, K. Xiao, A. Belianinov, and O. S. Ovchinnikova, Sci. Rep. 6, 30481 (2016).

${ }^{25}$ M. G. Stanford, P. R. Pudasaini, A. Belianinov, N. Cross, J. H. Noh, M. R. Koehler, D. G. Mandrus, G. Duscher, A. J. Rondinone, I. N. Ivanov, T. Z. Ward, and P. D. Rack, Sci. Rep. 6, 27276 (2016).

${ }^{26}$ M. G. Stanford, P. R. Pudasaini, E. T. Gallmeier, N. Cross, L. Liang, A. Oyedele, G. Duscher, M. Mahjouri-Samani, K. Wang, K. Xiao, D. B. Geohegan, A. Belianinov, B. G. Sumpter, and P. D. Rack, Adv. Funct. Mater. 27, 1702829 (2017).

${ }^{27}$ G. Nanda, G. Hlawacek, S. Goswami, K. Watanabe, T. Taniguchi, and P. F. A. Alkemade, Carbon 119, 419 (2017).
${ }^{28}$ M. O'Brien, N. McEvoy, T. Hallam, H. Y. Kim, N. C. Berner, D. Hanlon, K. Lee, J. N. Coleman, and G. S. Duesberg, Sci. Rep. 4, 7374 (2014).

${ }^{29}$ A. Castellanos-Gomez, M. Barkelid, A. M. Goossens, V. E. Calado, H. S. J. Van Der Zant, and G. A. Steele, Nano Lett. 12, 3187 (2012).

${ }^{30}$ L. Liang, J. Zhang, B. G. Sumpter, Q. H. Tan, P. H. Tan, and V. Meunier, ACS Nano 11, 11777 (2017)

${ }^{31}$ K. Liu, L. Zhang, T. Cao, C. Jin, D. Qiu, Q. Zhou, A. Zettle, P. Yang, S. G. Louie, and F. Wang, Nat. Commun. 5, 4966 (2014).

${ }^{32}$ S. Mignuzzi, A. J. Pollard, N. Bonini, B. Brennan, I. S. Gilmore, M. A. Pimenta, D. Richards, and D. Roy, Phys. Rev. B 91, 195411 (2015).

${ }^{33}$ J. Klein, A. Kuc, A. Nolinder, M. Altzschner, J. Wierzbowski, F. Sigger, F. Kreupl, J. J. Finley, U. Wurstbauer, A. W. Holleitner, and M. Kaniber, 2D Mater. 5, 011007 (2018).

${ }^{34}$ A. Splendiani, L. Sun, Y. Zhang, T. Li, J. Kim, C. Y. Chim, G. Galli, and F. Wang, Nano Lett. 10, 1271 (2010).

${ }^{35} \mathrm{~N}$. Scheuschner, O. Ochedowski, A. M. Kaulitz, R. Gillen, M. Schleberger, and J. Maultzsch, Phys. Rev. B 89, 125406 (2014).

${ }^{36}$ P. H. Tan, W. P. Han, W. J. Zhao, Z. H. Wu, K. Chang, H. Wang, Y. F. Wang, N. Bonini, N. Marzari, N. Pugno, G. Savini, A. Lombardo, and A. C. Ferrari, Nat. Mater. 11, 294 (2012).

${ }^{37}$ S. Kretschmer, M. Maslov, S. Ghaderzadeh, M. Ghorbani-Asl, G. Hlawacek, and A. V. Krasheninnikov, ACS Appl. Mater. Interfaces 10, 30827 (2018)

${ }^{38}$ W. M. Parkin, A. Balan, L. Liang, P. M. Das, M. Lamparski, C. H. Naylor, J. A. Rodríguez-Manzo, A. T. C. Johnson, V. Meunier, and M. Drndić, ACS Nano 10, 4134 (2016).

${ }^{39} \mathrm{~S}$. Huang, L. Liang, X. Ling, A. A. Puretzky, D. B. Geohegan, B. G. Sumpter, J. Kong, V. Meunier, and M. S. Dresselhaus, Nano Lett. 16, 1435 (2016).

${ }^{40}$ L. Liang, A. A. Puretzky, B. G. Sumpter, and V. Meunier, Nanoscale 9, 15340 (2017).

${ }^{41}$ I. Lahouij, B. Vacher, J. M. Martin, and F. Dassenoy, Wear 296, 558 (2012).

${ }^{42}$ C. Rueden, J. Schindelin, M. Hiner, B. DeZonia, A. Walter, E. Arena, and K. Eliceiri, BMC Bioinformatics 18, 1 (2017). 